\title{
Memorization vs. Generalization: Quantifying Data Leakage in NLP Performance Evaluation
}

\author{
Aparna Elangovan ${ }^{1}$, Jiayuan $\mathrm{He}^{1,2}$, and Karin Verspoor ${ }^{1}$ \\ ${ }^{1}$ The University of Melbourne, Australia \\ ${ }^{2}$ RMIT University, Australia \\ aparnae@student.unimelb.edu.au \\ \{estrid.he, karin.verspoor\}eunimelb.edu.au
}

\begin{abstract}
Public datasets are often used to evaluate the efficacy and generalizability of state-of-the-art methods for many tasks in natural language processing (NLP). However, the presence of overlap between the train and test datasets can lead to inflated results, inadvertently evaluating the model's ability to memorize and interpreting it as the ability to generalize. In addition, such data sets may not provide an effective indicator of the performance of these methods in real world scenarios. We identify leakage of training data into test data on several publicly available datasets used to evaluate NLP tasks, including named entity recognition and relation extraction, and study them to assess the impact of that leakage on the model's ability to memorize versus generalize.
\end{abstract}

\section{Introduction}

Shared tasks that provide publicly available datasets in order to evaluate and compare the performance of different methods on the same task and data are common in NLP. Held-out test sets are typically provided, enabling assessment of the generalizability of different methods to previously unseen data. These datasets have played a key role in driving progress in NLP, by defining focus tasks and by making annotated data available to the broader community, in particular in specialized domains such as biomedicine where data can be difficult to obtain, and quality data annotations require the detailed work of domain experts. Examples of tasks where benchmark data sets exist include open domain question answering (QA) (Berant et al., 2013; Joshi et al., 2017) and biomedical named entity recognition (Smith et al., 2008) .

In the context of machine learning models, effectiveness is typically determined by the model's ability to both memorize and generalize (Chatterjee, 2018). A model that has huge capacity to memo- rize will often work well in real world applications, particularly where large amounts of training data are available (Daelemans et al., 2005). The ability of a model to generalize relates to how well the model performs when it is applied on data that may be different from the data used to train the model, in terms of e.g. the distribution of vocabulary or other relevant vocabulary. The ability to memorize, taken to the extreme, can be considered equivalent to an exact match lookup table (Chatterjee, 2018) and the ability to generalize captures how well it can deal with degrees of variations from the lookup table. An effective combination of memorization and generalization can be achieved where a model selectively memorizes only those aspects or features that matter in solving a target objective given an input, allowing it to generalize better and to be less susceptible to noise.

When there is considerable overlap in the training and test data for a task, models that memorize more effectively than they generalize may benefit from the structure of the evaluation data, with their performance inflated relative to models that are more robust in generalization. However, such models may make poor quality predictions outside of the shared task setting. The external validity of these evaluations can therefore be questioned (Ferro et al., 2018).

In this paper, we assess the overlap between the train and test data in publicly available datasets for Named Entity Recognition (NER), Relation Extraction (REL) and Text Classification (CLS) tasks, including SST2 (Socher et al., 2013), BioCreative (Smith et al., 2008; Arighi et al., 2011) and AIMed (Bunescu et al., 2005) datasets, and examine the significant impact of not taking into account this overlap on performance evaluation.

We argue that robustness in generalization to unseen data is a key consideration of the performance of a model, and propose a framework to examine 
inadvertent leakage of data between data set splits, in order to enable more controlled assessment of the memorization vs. generalization characteristics of different methods.

\section{Related work}

The issue of memorization vs. generalization has been previously discussed in the context of question answering datasets, where, given only a question, a system must output the best answer it can find in available texts.

Lewis et al. (2020) identify 3 distinct issues for open domain QA evaluation: a) question memorization - recall the answer to a question that the model has seen at training time; b) answer memorization - answer novel questions at test time, where the model has seen the answer during training; and c) generalization - question and answer not seen during training time. They find that $58-71 \%$ of test answers occur in the training data in 3 examined data sets, concluding that the majority of the test data does not assess answer generalization. They also find that $28-34 \%$ have paraphrased questions in training data, and a majority of questions are duplicates differing only by a few words.

Similarly, Min (2020) identified repeating forms in QA test sets as a problem. The work proposed a novel template-based approach to splitting questions into paraphrase groups referred to as "Templates" and then controlling train/test data splits to ensure that all questions conforming to a given template appear in one segment of the data only. This was tested on the EMR Clinical Question Answering dataset emrQA (Pampari et al., 2018) and the Overnight dataset (Wang et al., 2015); it was demonstrated that models perform significantly worse on test sets where strict division is enforced. This paraphrase-based splitting methodology was also employed in their recent work on emrQA (Rawat et al., 2020).

\section{Approach}

A common practice to create a train and test set is to shuffle data instances in a dataset and generate random splits, without taking into account broader context. However, this can inadvertently lead to data leakage from the train set to test set due to the overlaps between similar train and test instances.

The type of overlap between train and test dataset depends on the type of the NLP task. Generally speaking, the leakage can occur either in the

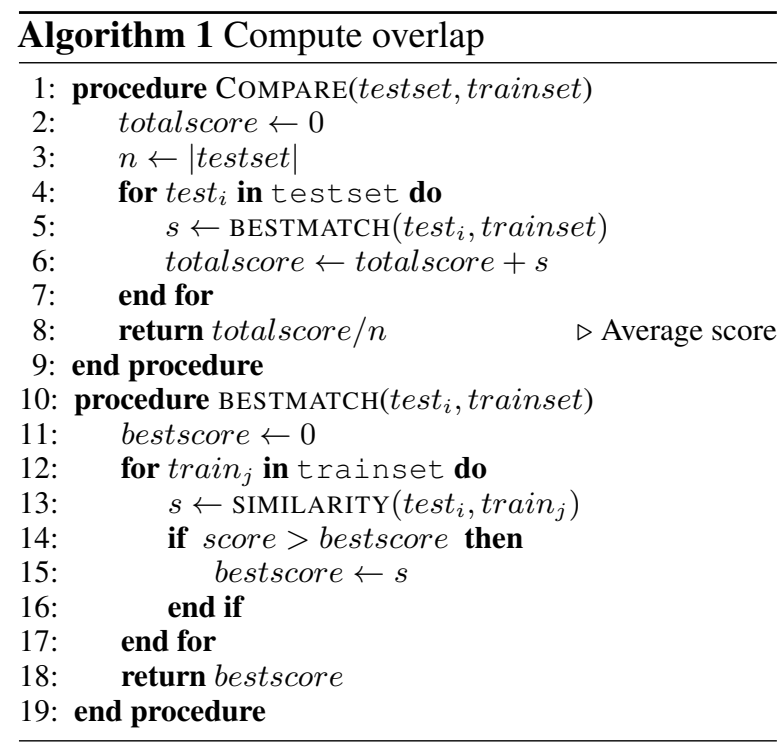

input texts or the annotated outputs. We define the types of overlaps which may occur in several NLP tasks as follows.

- In text classification (CLS) tasks such as sentiment analysis, overall (document-level) similarity in input texts can result in train/test leakage.

- In named entity recognition (NER) tasks, leakage from train to test data may occur when

a) input sentences or passages are similar

b) target entities are similar

- In relation extraction (REL) tasks, leakage may occur when

a) input sentences or passages are similar

b) participating entities are similar

We propose a framework for quantifying traintest overlaps, and conduct experiments to show the impact of train-test overlap on model performances. Next, we discuss the proposed framework in Sec. 4.2 and the experimental settings in Sec. 4.3. We present our findings including the train-test overlaps in several benchmark datasets in Sec. 5.1 and the impact of data leakage in Sec. 5.2.

\section{Method}

\subsection{Datasets}

We examine overlap in the following datasets:

- AIMed - AIMed dataset (Bunescu et al., 2005) for protein relation extraction (REL)

- BC2GM - BioCreative II gene mention dataset (Smith et al., 2008) for NER task

- ChEMU - Chemical Reactions from Patents (He et al., 2020) for recognising names of chemicals, an NER task 


\begin{tabular}{|c|c|c|c|c|}
\hline Task & Dataset & Score & Split & Example \\
\hline REL & AIMed (R) & 100.0 & Train & $\begin{array}{l}\text { Thus, during PROTEIN1 -mediated suppression of cell proliferation, PROTEIN } \\
\text { and PROTEIN } 2 \text { may be important for coordinating cell-cycle progression, DNA } \\
\text { replication and repair of damaged DNA. }\end{array}$ \\
\hline \multirow[t]{2}{*}{ CLS } & SST2 & 100.0 & Train & good movie. \\
\hline & & & Test & it 's still not a good movie. \\
\hline CLS & SST2 & 21.8 & Train & $\begin{array}{l}\text { herzog is obviously looking for a moral to his fable, but the notion that a strong, } \\
\text { unified showing among germany and eastern european jews might have changed } \\
\text { 20th-century history is undermined by ahola 's inadequate performance. } \\
\text { of the unsung heroes of 20th century }\end{array}$ \\
\hline
\end{tabular}

Table 1: Examples of train-test matches and the corresponding unigram similarity score.

- BC3ACT - Biocreative III protein interaction classification (CLS) (Arighi et al., 2011)

- SST2 - Stanford Sentiment Analysis Treebank (Socher et al., 2013) used to classify sentiments (CLS) in Glue (Wang et al., 2018)

The AIMed dataset does not explicitly provide a test set and 10-fold cross validation is used for evaluation in previous works (Hsieh et al., 2017; Zhang et al., 2019). In this paper, we use two types of splits of AIMed to evaluate the impact of data leakage: AIMed (R) which Randomly splits the dataset into 10 folds; and AIMed (U) which splits the dataset into 10 folds such that the documents within each resultant split are Unique (according to the document ID) to other splits across each split. The document ID refers to the source document of a data instance, and data instances from the same source document have the same document ID, see example in Appendix A

\subsection{Similarity measurement}

The pseudo code for measuring similarity is shown in Algorithm 1. Given a test instance test $t_{i}$, we compute its similarity with the training set using the training instance that is most similar with test ${ }_{i}$. We then use the average similarity over all the test instances as an indicator to measure the extent of train/test overlap. The function similarity $(\cdot)$ can be any function for text similarity. In this paper, we use a simple bag-of-words approach to compute text similarity. We represent each train/test instance with a count vector of unigrams/bigrams/trigrams, ignoring stopwords, and compute the similarity using the cosine similarity.

\subsection{Evaluate model performance}

We assess the impact of data leakage on a machine learning model's performance. We split the test sets of BC2GM, ChEMU, BC2ACT and SST2 into four intervals considering four similarity threshold ranges (in terms of unigrams): [0-0.25), [0.25$0.50),[0.50-0.75)$, and [0.75-1.0]. For example, the test instances in the first interval are most different from the training set with a similarity less than 0.25 . This method allows full control of the similarity of instances within each interval, but results in a different number of instances in each interval. Thus, we consider another scenario where we split the test set into 4 quartiles based on similarity ranking, so that the number of samples remain the same in each quartile but the threshold varies as a result.

We finetune a BERT (base and cased) model (Devlin et al., 2019) for each dataset using their own training set and compare the performance of the finetuned BERT model on the four different test intervals and test quartiles.

We compare the performances of AIMed (R) with AIMed (U) using 3 different models-Zhang et al. (2019) convolutional residual network, Hsieh et al. (2017) Bi-LSTM, and BioBERT (Lee et al., 2019). Following previous works, we preprocess the dataset and replace all non-participating proteins with neutral name PROTEIN, the participating entity pairs with PROTEIN1 and PROTEIN2, so the model only ever sees the pseudo protein names.

\section{Results}

\subsection{Similarity in datasets}

Examples of similar train and test instances are shown in Table 1. The overall results of train-test similarities of all datasets are shown in Table 2.

In the BC2GM dataset, we find that there is $70 \%$ overlap between gene names in the train and test set. On further analysis, we find that 2,308 out of 6,331 genes in the test set have exact matches in the train set. In the AIMed (R) dataset, we can see that there is over $73 \%$ overlap, even measured in 


\begin{tabular}{llrrr}
\hline Dataset & Task & uni & bi & tri \\
\hline AIMED (R) & REL & $\mathbf{9 6 . 9 5}$ & $\mathbf{8 2 . 2 9}$ & $\mathbf{7 3 . 1 5}$ \\
AIMED (U) & REL & $\mathbf{6 7 . 1 4}$ & 36.07 & 20.77 \\
BC2GM ann & NER & $\mathbf{7 0 . 7 7}$ & 19.55 & 5.41 \\
BC2GM text & NER & 33.19 & 13.12 & 4.20 \\
BC3ACT & CLS & 26.76 & 6.91 & 1.81 \\
ChEMU ann & NER & $\mathbf{8 4 . 2 9}$ & 30.67 & 6.83 \\
ChEMU text & NER & $\mathbf{6 8 . 4 5}$ & 42.39 & 31.63 \\
SST2 & CLS & 46.06 & 17.38 & 1.39 \\
\hline
\end{tabular}

Table 2: Train-test similarity using unigrams (uni), bigrams (bi), trigrams (tri). BC2GM and ChEMU are in BRAT standoff format and their similarities are shown for their text files ("text") and annotation files ("ann"). Similarities beyond 60.0 are highlighed in bold.

\begin{tabular}{llrrr}
\hline Split type & Method & P & R & F1 \\
\hline O & BiLSTM & 78.8 & 75.2 & 76.9 \\
O & ConvRes & 79.0 & 76.8 & 77.6 \\
\hline \multicolumn{4}{c}{ Replicated experiments } \\
\hline R & BiLSTM & 74.5 & 69.7 & 71.7 \\
U & BiLSTM & $\mathbf{5 7 . 4}$ & $\mathbf{6 1 . 7}$ & $\mathbf{5 8 . 7}$ \\
R & ConvRes & 71.1 & 69.2 & 69.9 \\
U & ConvRes & $\mathbf{5 6 . 7}$ & $\mathbf{5 6 . 4}$ & $\mathbf{5 6 . 1}$ \\
R & BioBERT & 79.8 & 76.7 & 77.9 \\
U & BioBERT & $\mathbf{6 5 . 8}$ & $\mathbf{6 3 . 7}$ & $\mathbf{6 4 . 4}$ \\
\hline
\end{tabular}

Table 3: Performances on AIMed (R) and AIMed (U). The split type $(\mathrm{O})$ indicates the original results from the authors.

the trigrams, between train and test sets.

\subsection{Model performance and similarity}

We observe drops in F-scores of more than 10 points between AIMed (R) and AIMed (U) across all three models as shown in Table 3. This is in line with the similarity measurement in Table 2: the train-test similarity drops significantly from AIMed (R) to AIMed (U) since in AIMed (U) we only allow unique document IDs in different folds.

On the ChEMU NER dataset we observe nearly 10-point drop in F-score $(96.7 \rightarrow 85.6)$ from 4 I to $2 \mathrm{I}$ as shown in Table 4.

On the BC2GM dataset, we also find that the model performance degrades from $82.4 \%$ to $74.5 \%$ in 2I compared to that in 1I. Surprisingly, Fscore for 4I is substantially lower than that of 3I $(78.5 \rightarrow 87.1)$, despite 41 out of the total 47 instances in 4 I having $100 \%$ similarity with the train set (full detailed samples shown in Appendix Table 10). A further investigation on this shows that (a) the interval $4 \mathrm{I}$ only has $0.9 \%(47 / 5000)$ of test instances; (b) a significant drop in recall

\begin{tabular}{llrrrrr}
\hline D & SR & \% & P & R & F1 & A \\
\hline BC2 & F & 100.0 & 77.5 & 86.4 & 81.7 & \\
BC2 & 1I & 19.8 & 68.8 & 81.1 & $\mathbf{7 4 . 5}$ & \\
BC2 & 2I & 74.1 & 78.3 & 86.9 & 82.4 & \\
BC2 & 3I & 5.1 & 83.8 & 90.6 & $\mathbf{8 7 . 1}$ & \\
BC2 & 4I & 0.9 & 79.5 & 77.5 & 78.5 & \\
\hline ChE & F & 100.0 & 93.8 & 94.4 & 94.1 & \\
ChE & 1I & 0.0 & - & - & - & \\
ChE & 2I & 10.0 & 84.6 & 86.6 & $\mathbf{8 5 . 6}$ & \\
ChE & 3I & $\mathbf{6 0 . 0}$ & 93.4 & 94.0 & 93.7 & \\
ChE & 4I & 30.0 & 96.7 & 96.7 & $\mathbf{9 6 . 7}$ & \\
\hline BC3 & F & 100.0 & 45.1 & 84.1 & 58.7 & 82.1 \\
BC3 & 1I & 47.0 & 43.0 & 82.0 & $\mathbf{5 6 . 4}$ & 85.8 \\
BC3 & 2I & 51.0 & 46.0 & 85.6 & 59.9 & 78.8 \\
BC3 & 3I & 2.0 & 53.5 & 76.7 & $\mathbf{6 3 . 0}$ & 77.5 \\
BC3 & 4I & 0.0 & 0.0 & 0.0 & 0.0 & 0.0 \\
\hline SST & F & 100.0 & 90.4 & 96.7 & 93.4 & 93.2 \\
SST & 1I & 1.1 & 60.0 & 75.0 & $\mathbf{6 6 . 7}$ & $\mathbf{8 5 . 0}$ \\
SST & 2I & 66.8 & 91.6 & 96.0 & 93.8 & 93.4 \\
SST & 3I & 28.7 & 87.1 & 98.7 & 92.5 & 92.7 \\
SST & 4 I & 3.5 & 96.9 & 96.9 & $\mathbf{9 6 . 9}$ & $\mathbf{9 6 . 8}$ \\
\hline
\end{tabular}

Table 4: Performances on various similarity thresholds and the corresponding percentage of test instances within the intervals. Datasets (D): $\mathrm{BC} 2 \rightarrow \mathrm{BC} 2 \mathrm{GM}$, $\mathrm{ChE} \rightarrow \mathrm{ChEMU}, \mathrm{BC} 3 \rightarrow \mathrm{BC} 3 \mathrm{ACT}, \mathrm{SST} \rightarrow \mathrm{SST} 2$. The similarity threshold range $(\mathrm{SR})[0,0.25)=1 \mathrm{I}$, $[0.25,0.5)=2 \mathrm{I},[0.5,0.75)=3 \mathrm{I},[0.75,1]=4 \mathrm{I}$, $[0,1]=$ F. Accuracy (A) is the official metric for the SST2 dataset according to GLUE benchmark, all others use $\mathrm{F} 1$-score $(\mathrm{F} 1)$ as primary metric.

$(90.6 \rightarrow 77.5)$ from $3 \mathrm{I}$ to $4 \mathrm{I}$ is caused by six instances whose input texts have exact matches in the train set (full samples shown in Appendix Table 11). This implies that the model doesn't perform well even on the training data for these samples. Since BC2GM has over 70\% overlap in the target gene mentions (Table 2), we also analysed the recall on the annotations that overlap between train and test. We find that the recall increases $(84.5 \rightarrow 87.8)$, see Appendix Table 8, compared to recall $(81.1 \rightarrow 90.6)$ as a result of input text similarity. Since BERT uses a word sequence-based prediction approach, the relatively high similarity in target annotations does not seem to make much difference compared to similarity in input text. However, if we used a dictionary-based approach, similarity in annotations could result in much higher recall compared to similarity in input text.

The BC3ACT dataset also exhibits the same trend where the F1-score improves $(56.4 \rightarrow 63.0)$ as the similarity increases. However, the accuracy 


\begin{tabular}{lcrrrrrr}
\hline D & Q & Min & Max & P & R & F1 & A \\
\hline BC2 & 1 & 0.0 & 26.3 & 69.8 & 82.0 & $\mathbf{7 5 . 4}$ & \\
BC2 & 2 & 26.3 & 31.6 & 74.5 & 85.9 & 79.8 & \\
BC2 & 3 & 31.6 & 38.3 & 78.3 & 86.4 & 82.1 & \\
BC2 & 4 & 38.3 & 100.0 & 83.0 & 88.9 & $\mathbf{8 5 . 9}$ & \\
\hline ChE & 1 & 37.9 & 56.7 & 90.8 & 91.8 & $\mathbf{9 1 . 3}$ & \\
ChE & 2 & 56.8 & 68.2 & 93.3 & 94.4 & 93.8 & \\
ChE & 3 & 68.2 & 78.5 & 95.1 & 96.1 & 95.6 & \\
ChE & 4 & 78.6 & 99.8 & 97.1 & 97.4 & $\mathbf{9 7 . 3}$ & \\
\hline BC3 & 1 & 6.3 & 20.1 & 44.5 & 81.4 & $\mathbf{5 7 . 6}$ & 88.8 \\
BC3 & 2 & 20.1 & 25.7 & 42.3 & 82.5 & 55.9 & 82.7 \\
BC3 & 3 & 25.7 & 31.9 & 46.5 & 85.3 & $\mathbf{6 0 . 2}$ & 79.5 \\
BC3 & 4 & 31.9 & 75.0 & 46.1 & 85.2 & 59.8 & 77.3 \\
\hline SST & 1 & 0.0 & 36.5 & 90.8 & 95.2 & $\mathbf{9 2 . 9}$ & $\mathbf{9 2 . 8}$ \\
SST & 2 & 36.5 & 43.6 & 91.3 & 96.2 & 93.7 & 93.2 \\
SST & 3 & 43.6 & 53.5 & 91.2 & 97.3 & $\mathbf{9 4 . 2}$ & $\mathbf{9 4 . 1}$ \\
SST & 4 & 53.5 & 100.0 & 88.0 & 98.1 & 92.8 & 92.9 \\
\hline
\end{tabular}

Table 5: Performances on four different test quartiles, where the number of samples in each quartile $(\mathrm{Q})$ is kept same. The minimum (Min) and the maximum (Max) similarity within each quartile are also reported.

drops from $85.8 \rightarrow 77.5$. This is could be because while the train set has $50 \%$ positive classes, the test set has just $17 \%$ with 3 points higher mean similarity in positive samples (details in Appendix B).

On SST2, an increase in accuracy $(85.0 \rightarrow 96.8)$ from 1I to 4I is observed apart from a marginal 0.7-point drop (93.4 $\rightarrow$ 92.7) from 2I to 3I.

We also split the test sets into four equal-sized quartiles based on the similarity ranking of test instances, shown in Table 5. We observe similar phenomena as in the previous set of experiments for the dataset BC2GM, ChEMU, and BC3ACT. The only exception is for SST2 where the F-score has a relatively small but consistent increase from Q1 to Q3 (92.9 $\rightarrow 94.1)$ but drops to 92.8 in Q4.

\section{Discussion}

\subsection{Quantifying similarity}

The bag-of-words based approach to compute cosine similarity has been able to detect simple forms of overlap effectively as shown in Table 2. A trend that can be seen is that overlap is more common in tasks that are manual labour intensive, such as named entity recognition and relation extraction compared to text classification.

However, this approach may detect similarity even when the meanings are different, especially in the case of classification tasks as shown for SST2 in Table 1. Semantic Text Similarity (STS) mea- surement is a challenging task in its own right, with a large body of literature and a number of shared tasks organized to address it (Cer et al., 2017; Wang et al., 2020; Karimi et al., 2015). More sophisticated methods for similarity measurement developed in these contexts could be incorporated into the framework for measuring similarity of data set splits; for simple leakage detection it is arguably adequate. However, sophisticated methods can also potentially lead to a chicken and egg problem, if we use a machine learning model to compute semantic similarity.

The question of what level of similarity is acceptable is highly data and task-dependent. If the training data has good volume and variety, the trainingtest similarity will naturally be higher and so will the acceptable similarity.

\subsection{Memorization vs. Generalization}

We find that the F-scores tend to be higher when the test set input text is similar to the training set as shown in Table 3 and 4. While this might be apparent, quantifying similarity in the test set helps understand that high scores in the test set could be a result of similarity to the train set, and therefore measuring memorization and not a model's ability to generalize. If a model is trained on sufficient volume and variety of data then it may now matter if it memorizes or generalizes in a real world context, and a model's ability to memorize is not necessarily a disadvantage. However, in the setting of a shared task, we often do not have access to sufficiently large training data sets and hence it is important to consider the test/train similarity when evaluating the models. This implies that in real world scenarios the model may perform poorly when it encounters data not seen during training.

\section{Conclusion}

We conclude that quantifying train/test overlap is crucial to assessing real world applicability of machine learning in NLP tasks, given our reliance on annotated data for training and testing in the NLP community. A single metric over a held-out test set is not sufficient to infer generalizablity of a model. Stratification of test sets by similarity enables more robust assessment of memorization vs. generalization capabilities of models. Further development of approaches to structured consideration of model performance under different assumptions will improve our understanding of these tradeoffs. 


\section{References}

Cecilia N Arighi, Phoebe M Roberts, Shashank Agarwal, Sanmitra Bhattacharya, Gianni Cesareni, Andrew Chatr-Aryamontri, Simon Clematide, Pascale Gaudet, Michelle Gwinn Giglio, Ian Harrow, et al. 2011. BioCreative III interactive task: an overview. BMC Bioinformatics, 12(S8):S4.

Jonathan Berant, Andrew Chou, Roy Frostig, and Percy Liang. 2013. Semantic parsing on Freebase from question-answer pairs. In Proceedings of the 2013 Conference on Empirical Methods in Natural Language Processing, pages 1533-1544. Association for Computational Linguistics.

Razvan Bunescu, Ruifang Ge, Rohit J Kate, Edward M Marcotte, Raymond J Mooney, Arun K Ramani, and Yuk Wah Wong. 2005. Comparative experiments on learning information extractors for proteins and their interactions. Artificial Intelligence in Medicine, 33(2):139-155.

Daniel Cer, Mona Diab, Eneko Agirre, Iñigo LopezGazpio, and Lucia Specia. 2017. SemEval-2017 Task 1: Semantic textual similarity multilingual and crosslingual focused evaluation. In Proceedings of the 11th International Workshop on Semantic Evaluation (SemEval-2017), pages 1-14, Vancouver, Canada. Association for Computational Linguistics.

Satrajit Chatterjee. 2018. Learning and memorization. In Proceedings of the 35th International Conference on Machine Learning, volume 80 of Proceedings of Machine Learning Research, pages 755-763, Stockholmsmässan, Stockholm Sweden. PMLR.

Walter Daelemans, Antal Van den Bosch, et al. 2005. Memory-based language processing. Cambridge University Press.

Jacob Devlin, Ming-Wei Chang, Kenton Lee, and Kristina Toutanova. 2019. BERT: Pre-training of deep bidirectional transformers for language understanding. In Proceedings of the 2019 Conference of the North American Chapter of the Association for Computational Linguistics: Human Language Technologies, Volume 1 (Long and Short Papers), pages 4171-4186, Minneapolis, Minnesota. Association for Computational Linguistics.

Nicola Ferro, Norbert Fuhr, Gregory Grefenstette, Joseph A Konstan, Pablo Castells, Elizabeth M Daly, Thierry Declerck, Michael D Ekstrand, Werner Geyer, Julio Gonzalo, et al. 2018. The dagstuhl perspectives workshop on performance modeling and prediction. In ACM SIGIR Forum, volume 52, pages 91-101. ACM New York, NY, USA.

Jiayuan He, Dat Quoc Nguyen, Saber A. Akhondi, Christian Druckenbrodt, Camilo Thorne, Ralph Hoessel, Zubair Afzal, Zenan Zhai, Biaoyan Fang, Hiyori Yoshikawa, Ameer Albahem, Lawrence Cavedon, Trevor Cohn, Timothy Baldwin, and Karin Verspoor. 2020. Overview of ChEMU 2020: Named entity recognition and event extraction of chemical reactions from patents. In Experimental IR Meets Multilinguality, Multimodality, and Interaction. Proceedings of the Eleventh International Conference of the CLEF Association (CLEF 2020), volume 12260. Lecture Notes in Computer Science.

Yu-Lun Hsieh, Yung-Chun Chang, Nai-Wen Chang, and Wen-Lian Hsu. 2017. Identifying proteinprotein interactions in biomedical literature using recurrent neural networks with long short-term memory. In Proceedings of the Eighth International Joint Conference on Natural Language Processing (Volume 2: Short Papers), pages 240-245, Taipei, Taiwan. Asian Federation of Natural Language Processing.

Mandar Joshi, Eunsol Choi, Daniel Weld, and Luke Zettlemoyer. 2017. TriviaQA: A large scale distantly supervised challenge dataset for reading comprehension. In Proceedings of the 55th Annual Meeting of the Association for Computational Linguistics (Volume 1: Long Papers), pages 1601-1611, Vancouver, Canada. Association for Computational Linguistics.

Sarvnaz Karimi, Jie Yin, and Jiri Baum. 2015. Evaluation methods for statistically dependent text. Computational Linguistics, 41(3):539-548.

Jinhyuk Lee, Wonjin Yoon, Sungdong Kim, Donghyeon Kim, Sunkyu Kim, Chan Ho So, and Jaewoo Kang. 2019. BioBERT: a pretrained biomedical language representation model for biomedical text mining. Bioinformatics, 36(4):1234-1240.

Patrick Lewis, Pontus Stenetorp, and Sebastian Riedel. 2020. Question and answer test-train overlap in open-domain question answering datasets.

So Yeon Min. 2020. Towards knowledge-based, robust question answering.

Anusri Pampari, Preethi Raghavan, Jennifer Liang, and Jian Peng. 2018. emrQA: A large corpus for question answering on electronic medical records. In Proceedings of the 2018 Conference on Empirical Methods in Natural Language Processing, pages 2357-2368, Brussels, Belgium. Association for Computational Linguistics.

Bhanu Pratap Singh Rawat, Wei-Hung Weng, So Yeon Min, Preethi Raghavan, and Peter Szolovits. 2020. Entity-enriched neural models for clinical question answering. In Proceedings of the 19th SIGBioMed Workshop on Biomedical Language Processing, pages 112-122, Online. Association for Computational Linguistics.

L Smith, LK Tanabe, RJ Ando, CJ Kuo, IF Chung, CN Hsu, YS Lin, R Klinger, CM Friedrich, $\mathrm{K}$ Ganchev, et al. 2008. Overview of BioCreative II gene mention recognition. Genome Biology, 9:S2S2. 
Richard Socher, Alex Perelygin, Jean Wu, Jason Chuang, Christopher D. Manning, Andrew Ng, and Christopher Potts. 2013. Recursive deep models for semantic compositionality over a sentiment treebank. In Proceedings of the 2013 Conference on Empirical Methods in Natural Language Processing, pages 1631-1642, Seattle, Washington, USA. Association for Computational Linguistics.

Alex Wang, Amanpreet Singh, Julian Michael, Felix Hill, Omer Levy, and Samuel Bowman. 2018. GLUE: A multi-task benchmark and analysis platform for natural language understanding. In Proceedings of the 2018 EMNLP Workshop BlackboxNLP: Analyzing and Interpreting Neural Networks for NLP, pages 353-355, Brussels, Belgium. Association for Computational Linguistics.

Yanshan Wang, Naveed Afzal, Sunyang Fu, Liwei Wang, Feichen Shen, Majid Rastegar-Mojarad, and Hongfang Liu. 2020. MedSTS: a resource for clinical semantic textual similarity. Language Resources and Evaluation, 54(1):57-72.

Yushi Wang, Jonathan Berant, and Percy Liang. 2015. Building a semantic parser overnight. In Proceedings of the 53rd Annual Meeting of the Association for Computational Linguistics and the 7th International Joint Conference on Natural Language Processing (Volume 1: Long Papers), pages 1332-1342, Beijing, China. Association for Computational Linguistics.

H. Zhang, R. Guan, F. Zhou, Y. Liang, Z. Zhan, L. Huang, and X. Feng. 2019. Deep residual convolutional neural network for protein-protein interaction extraction. IEEE Access, 7:89354-89365. 


\section{A AIMed document examples}

The following example shows how multiple data instances are extracted from a single document in AIMed dataset. The document with ID "AIMed.d0" has several instances including "AIMed.d0.s0" and "AIMed.d0.s1". These instances thus have the same document id.

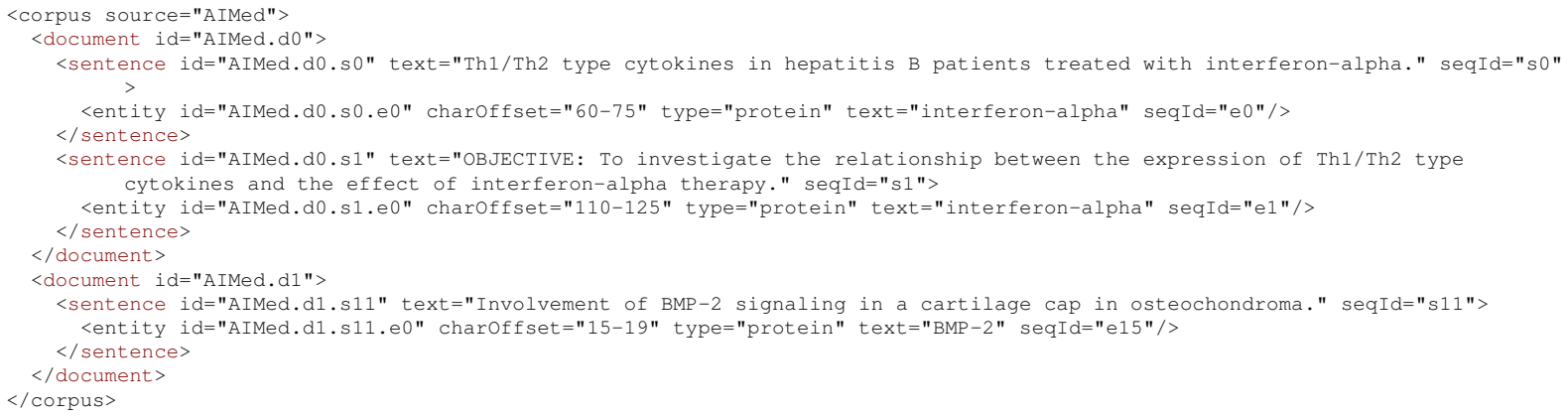

\section{B Classwise similarity for BC3AST}

The test set has 5090 negative samples compared to 910 positive samples, with 2.96 points higher mean similarity in positive samples.

\begin{tabular}{llrrr}
\hline Test label & & Unigram & Bigram & Trigram \\
\hline 0 & count & 5090.00 & 5090.00 & 5090.00 \\
& mean & 26.31 & 6.70 & 1.73 \\
& std & 9.25 & 5.35 & 1.72 \\
min & 6.28 & 0.00 & 0.00 \\
& $25 \%$ & 19.70 & 3.29 & 0.79 \\
& $50 \%$ & 25.16 & 5.07 & 1.39 \\
& $75 \%$ & 31.53 & 8.29 & 2.27 \\
& max & 75.01 & 41.75 & 18.71 \\
1 & count & 910.00 & 910.00 & 910.00 \\
& mean & 29.27 & 8.09 & 2.26 \\
& std & 9.36 & 6.00 & 1.73 \\
& min & 11.14 & 1.52 & 0.00 \\
& $25 \%$ & 22.69 & 4.51 & 1.17 \\
& $50 \%$ & 28.31 & 6.25 & 1.88 \\
& $75 \%$ & 34.32 & 9.38 & 2.84 \\
& max & 74.01 & 51.20 & 18.97 \\
\hline
\end{tabular}

Table 6: Class-wise similarity for BC3ACT dataset

\section{BERT and similarity thresholds}

Table 7 shows the impact on precision, recall and F-score using different similarity thresholds on the BC2GM test set, which has approximately 6,300 annotations.

We also compare the recall when the target annotations are similar as shown in Table 8. We only compare unigrams, as the number of tokens in a gene name tends to be small (on average less than 3).

Table 9 shows BERT's performance using bi-grams and trigrams on SST2 and BC3AST datasets. 


\begin{tabular}{lrrrrrr}
\hline Dataset & N & SR & $\%$ & P & R & F \\
\hline BC2GM & - & - & 100 & 77.5 & 86.4 & 81.7 \\
\hline BC2GM & 1 & 1I & 19.8 & 68.8 & 81.1 & 74.5 \\
BC2GM & 1 & 2I & 74.1 & 78.2 & 86.9 & 82.3 \\
BC2GM & 1 & 3I & 5.1 & 83.8 & 90.6 & 87.1 \\
BC2GM & 1 & 4I & 1.0 & 79.5 & 77.5 & 78.5 \\
\hline BC2GM & 2 & 1I & 91.7 & 76.9 & 86.3 & 81.4 \\
BC2GM & 2 & 2I & 7.5 & 82.5 & 88.1 & 85.2 \\
BC2GM & 2 & 3I & 0.3 & 1.0 & 1.0 & 1.0 \\
BC2GM & 2 & 4I & 0.5 & 78.9 & 76.9 & 77.9 \\
\hline BC2GM & 3 & 1I & 98.5 & 77.4 & 86.4 & 81.7 \\
BC2GM & 3 & 2I & 0.9 & 85.2 & 88.5 & 86.8 \\
BC2GM & 3 & 3I & 0.1 & 50.0 & 100.0 & 66.7 \\
BC2GM & 3 & 4I & 0.5 & 80.6 & 76.3 & 78.4 \\
\hline
\end{tabular}

Table 7: NER performances of BERT on various similarity threshold range (SR) and the corresponding percentage of instances when the similarity is computed using $\mathrm{N}$-grams $(\mathrm{N}=1,2$ and 3$)$ in the input text. The range $[0,0.25)=$ $1 \mathrm{I},[0.25,0.5)=2 \mathrm{I},[0.5,0.75)=3 \mathrm{I},[0.75,1]=4 \mathrm{I},[0,1]=\mathrm{F}$.

\begin{tabular}{lclrr}
\hline Dataset & N & SR & $\%$ & Recall \\
\hline BC2GM (anno) & - & F & 100.0 & 86.4 \\
BC2GM (anno) & 1 & 1I & 16.7 & 84.5 \\
BC2GM (anno) & 1 & 2I & 5.6 & 81.8 \\
BC2GM (anno) & 1 & 3I & 24.7 & 85.6 \\
BC2GM (anno) & 1 & 4I & 53.0 & 87.8 \\
\hline
\end{tabular}

Table 8: NER score on BERT at various similarity threshold range (SR) and the corresponding \% of samples using ngram $\mathrm{N}=1$ in the output annotated gene mentions.

\begin{tabular}{lclrrrrr}
\hline Dataset & N & SR & $\%$ & $\mathrm{P}$ & $\mathrm{R}$ & $\mathrm{F} 1$ & $\mathrm{~A}$ \\
\hline BC3ACT & - & F & 100.0 & 45.1 & 84.1 & 58.7 & 82.1 \\
BC3ACT & 1 & 1I & 47.0 & 43.0 & 82.0 & 56.4 & 85.8 \\
BC3ACT & 1 & 2I & 51.0 & 46.0 & 85.6 & 59.9 & 78.8 \\
BC3ACT & 1 & 3I & 2.0 & 53.5 & 76.7 & 63.0 & 77.5 \\
BC3ACT & 1 & 4I & 0.0 & 0.0 & 0.0 & 0.0 & 0.0 \\
BC3ACT & 2 & 1I & 98.2 & 45.0 & 84.1 & 58.6 & 82.2 \\
BC3ACT & 2 & 2I & 1.8 & 48.8 & 83.3 & 61.5 & 76.6 \\
BC3ACT & 2 & 3I & 0.0 & 100.0 & 100.0 & 100.0 & 100.0 \\
BC3ACT & 2 & 4I & 0.0 & 0.0 & 0.0 & 0.0 & - \\
BC3ACT & 3 & 1I & 100.0 & 45.1 & 84.1 & 58.7 & 82.1 \\
BC3ACT & 3 & 2I & 0.0 & 0.0 & 0.0 & 0.0 & - \\
BC3ACT & 3 & 3I & 0.0 & 0.0 & 0.0 & 0.0 & - \\
BC3ACT & 3 & 4I & 0.0 & 0.0 & 0.0 & 0.0 & - \\
\hline SST2 & - & F & 100.0 & 90.4 & 96.7 & 93.4 & 93.2 \\
SST2 & 1 & 1I & 1.1 & 60.0 & 75.0 & 66.7 & 85.0 \\
SST2 & 1 & 2I & 66.8 & 91.6 & 96.0 & 93.8 & 93.4 \\
SST2 & 1 & 3I & 28.7 & 87.1 & 98.7 & 92.5 & 92.7 \\
SST2 & 1 & 4I & 3.5 & 96.9 & 96.9 & 96.9 & 96.8 \\
SST2 & 2 & 1I & 64.0 & 88.6 & 96.0 & 92.2 & 92.3 \\
SST2 & 2 & 2I & 30.8 & 93.1 & 97.3 & 95.1 & 94.8 \\
SST2 & 2 & 3I & 4.8 & 93.1 & 100.0 & 96.4 & 95.4 \\
SST2 & 2 & 4I & 0.4 & 100.0 & 100.0 & 100.0 & 100.0 \\
SST2 & 3 & 1I & 97.6 & 90.5 & 96.6 & 93.4 & 93.3 \\
SST2 & 3 & 2I & 1.9 & 82.6 & 100.0 & 90.5 & 88.2 \\
SST2 & 3 & 3I & 0.5 & 100.0 & 100.0 & 100.0 & 100.0 \\
SST2 & 3 & 4I & 0.0 & 0.0 & 0.0 & 0.0 & - \\
\hline & & & & & & &
\end{tabular}

Table 9: SST2 and BC3ACT similarity thresholds using ngram $\mathrm{N}=1,2$ and 3 . The range $[0,0.25)=1 I$, $[0.25,0.5)=2 I,[0.5,0.75)=3 I,[0.75,1]=4 I,[0,1]=F$ 


\section{High similarity BC2GM samples}

Table 10 shows the $75 \%$ similarity samples in the $\mathrm{BC} 2 \mathrm{GM}$ dataset. The samples that caused the drop in recall are shown in Table 11.

\begin{tabular}{|c|c|c|}
\hline Score & Test & Train \\
\hline 76.45 & $\begin{array}{l}\text { Histological and immunophenotypic studies revealed } 12 \text { large cell lymphomas (11 B } \\
\text { cell and one T cell), two small noncleaved cell lymphomas (B-cell phenotype), and } \\
\text { five low grade B-cell lymphomas (two small lymphocytic and three follicular mixed } \\
\text { lymphomas). }\end{array}$ & $\begin{array}{l}\text { The cases included } 35 \text { de novo diffuse aggressive lymphomas (DAL; } 19 \text { large-cell, } 4 \\
\text { mixed-cell, and } 12 \text { large-cell immunoblastic), } 52 \text { transformed aggressive lymphomas } \\
\text { derived from follicular lymphomas (TFL), } 42 \text { indolent follicular lymphomas (FL), } 14 \\
\text { mantle cell lymphomas (MCL), and } 27 \text { small noncleaved cell lymphomas (SNCL). }\end{array}$ \\
\hline 77.46 & $98,93-98)$ & $356,93-98]$. \\
\hline 81.65 & Free protein $\mathrm{S}$ deficiency in acute ischemic stroke. & Ischemic stroke due to protein $\mathrm{C}$ deficiency. \\
\hline 83.41 & $\begin{array}{l}\text { In stage I, histochemistry for copper was positive in } 11 \text { out of } 21 \text { cases: } 6 \text { cases were } \\
T+; 1 \text { case } R+\text { and } 2 \text { cases } O+; 2 \text { cases were } T+, R+, O+\text {. }\end{array}$ & 3 cases \\
\hline 86.60 & STUDY DESIGN: Retrospective review. & DESIGN: Retrospective study. \\
\hline 86.60 & Non-dialyzable transfer factor & Dialyzable transfer factor. \\
\hline 100.00 & $\begin{array}{l}\text { Recently we have performed a detailed analysis of specific neuronal populations af- } \\
\text { fected by the mutation which shed new light on the role of Krox- } 20 \text { in the segmentation } \\
\text { and on the physiological consequences of its inactivation. }\end{array}$ & $\begin{array}{l}\text { Recently we have performed a detailed analysis of specific neuronal populations af- } \\
\text { fected by the mutation which shed new light on the role of Krox- } 20 \text { in the segmentation } \\
\text { and on the physiological consequences of its inactivation. }\end{array}$ \\
\hline 100.00 & $\begin{array}{l}\text { Slowly adapting type I mechanoreceptor discharge as a function of dynamic force } \\
\text { versus dynamic displacement of glabrous skin of raccoon and squirrel monkey hand. }\end{array}$ & $\begin{array}{l}\text { Slowly adapting type I mechanoreceptor discharge as a function of dynamic force } \\
\text { versus dynamic displacement of glabrous skin of raccoon and squirrel monkey hand. }\end{array}$ \\
\hline 100.00 & $\begin{array}{l}\text { The recruitment of constitutively phosphorylated } \mathrm{p} 185(\mathrm{neu}) \text { and the activated mito- } \\
\text { genic pathway proteins to this membrane-microfilament interaction site provides a } \\
\text { physical model for integrating the assembly of the mitogenic pathway with the trans- } \\
\text { mission of growth factor signal to the cytoskeleton. }\end{array}$ & $\begin{array}{l}\text { The recruitment of constitutively phosphorylated } \mathrm{p} 185(\mathrm{neu}) \text { and the activated mito- } \\
\text { genic pathway proteins to this membrane-microfilament interaction site provides a } \\
\text { physical model for integrating the assembly of the mitogenic pathway with the trans- } \\
\text { mission of growth factor signal to the cytoskeleton. }\end{array}$ \\
\hline 100.00 & $\begin{array}{l}\text { A heterologous promoter construct containing three repeats of a consensus Sp1 site, } \\
\text { cloned upstream of a single copy of the ZII (CREB/ AP1) element from the BZLF1 } \\
\text { promoter linked to the beta-globin TATA box, exhibited phorbol ester inducibility. }\end{array}$ & $\begin{array}{l}\text { A heterologous promoter construct containing three repeats of a consensus Sp1 site, } \\
\text { cloned upstream of a single copy of the ZII (CREB/ AP1) element from the BZLF1 } \\
\text { promoter linked to the beta-globin TATA box, exhibited phorbol ester inducibility. }\end{array}$ \\
\hline 100.00 & $\begin{array}{l}\text { The reconstituted RNA polymerases containing the mutant alpha subunits were exam- } \\
\text { ined for their response to transcription activation by cAMP-CRP and the rrnBP1 UP } \\
\text { element. }\end{array}$ & $\begin{array}{l}\text { The reconstituted RNA polymerases containing the mutant alpha subunits were exam- } \\
\text { ined for their response to transcription activation by cAMP-CRP and the rrnBP1 UP } \\
\text { element. }\end{array}$ \\
\hline 100.00 & $\begin{array}{l}\text { Analysis of } 1 \mathrm{Mb} \text { of published sequence from the region of conserved synteny on } \\
\text { human chromosome } 5 \mathrm{q} 31-\mathrm{q} 33 \text { identified } 45 \text { gene candidates, including } 35 \text { expressed } \\
\text { genes in the human IL- } 4 \text { cytokine gene cluster. }\end{array}$ & $\begin{array}{l}\text { Analysis of } 1 \mathrm{Mb} \text { of published sequence from the region of conserved synteny on } \\
\text { human chromosome } 5 \mathrm{q} 31 \text { - } \mathrm{q} 33 \text { identified } 45 \text { gene candidates, including } 35 \text { expressed } \\
\text { genes in the human IL- } 4 \text { cytokine gene cluster. }\end{array}$ \\
\hline 100.00 & $\begin{array}{l}\text { Although RAD17, RAD24 and MEC3 are not required for cell cycle arrest when S } \\
\text { phase is inhibited by hydroxyurea (HU), they do contribute to the viability of yeast } \\
\text { cells grown in the presence of HU, possibly because they are required for the repair of } \\
\text { HU-induced DNA damage. }\end{array}$ & $\begin{array}{l}\text { Although RAD17, RAD24 and MEC3 are not required for cell cycle arrest when S } \\
\text { phase is inhibited by hydroxyurea (HU), they do contribute to the viability of yeast } \\
\text { cells grown in the presence of HU, possibly because they are required for the repair of } \\
\text { HU-induced DNA damage. }\end{array}$ \\
\hline 100.00 & $\begin{array}{l}\text { The promoter for HMG-CoA synthase contains two binding sites for the sterol regula- } \\
\text { tory element-binding proteins (SREBPs). }\end{array}$ & $\begin{array}{l}\text { The promoter for HMG-CoA synthase contains two binding sites for the sterol regula- } \\
\text { tory element-binding proteins (SREBPs). }\end{array}$ \\
\hline 100.00 & $\begin{array}{l}\text { Coronary vasoconstriction caused by endothelin- } 1 \text { is enhanced by ischemia- } \\
\text { reperfusion and by norepinephrine present in concentrations typically observed after } \\
\text { neonatal cardiopulmonary bypass. }\end{array}$ & $\begin{array}{l}\text { Coronary vasoconstriction caused by endothelin- } 1 \text { is enhanced by ischemia- } \\
\text { reperfusion and by norepinephrine present in concentrations typically observed after } \\
\text { neonatal cardiopulmonary bypass. }\end{array}$ \\
\hline 100.00 & $($ LH P $; 0.05$, LH/FSH P $; 0.01)$ & $($ LH P $; 0.05$, LH/FSH P $; 0.01)$ \\
\hline 100.00 & $\begin{array}{l}\text { Determinants of recurrent ischaemia and revascularisation procedures after thrombol- } \\
\text { ysis with recombinant tissue plasminogen activator in primary coronary occlusion. }\end{array}$ & $\begin{array}{l}\text { Determinants of recurrent ischaemia and revascularisation procedures after thrombol- } \\
\text { ysis with recombinant tissue plasminogen activator in primary coronary occlusion. }\end{array}$ \\
\hline 100.00 & $\begin{array}{l}\text { The human SHBG proximal promoter was analyzed by DNase I footprinting, and } \\
\text { the functional significance of } 6 \text { footprinted regions (FP1-FP6) within the proximal } \\
\text { promoter was studied in human HepG2 hepatoblastoma cells. }\end{array}$ & $\begin{array}{l}\text { The human SHBG proximal promoter was analyzed by DNase I footprinting, and } \\
\text { the functional significance of } 6 \text { footprinted regions (FP1-FP6) within the proximal } \\
\text { promoter was studied in human HepG } 2 \text { hepatoblastoma cells. }\end{array}$ \\
\hline 100.00 & Biol. & Biol. \\
\hline 100.00 & Copyright 1999 Academic Press. & Copyright 1999 Academic Press. \\
\hline 100.00 & $\begin{array}{l}\text { These results demonstrate a specific association of SIV and HIV-2 nef, but not HIV-1 } \\
\text { nef, with TCRzeta. }\end{array}$ & $\begin{array}{l}\text { These results demonstrate a specific association of SIV and HIV-2 nef, but not HIV-1 } \\
\text { nef, with TCRzeta. }\end{array}$ \\
\hline 100.00 & $\begin{array}{l}\text { Urease activity, judged as the amount of ammonia production from urea, could be } \\
\text { measured at } 25 \text { ng per tube }(\mathrm{S} / \mathrm{N}=1.5) \text { with Jack bean meal urease. }\end{array}$ & $\begin{array}{l}\text { Urease activity, judged as the amount of ammonia production from urea, could be } \\
\text { measured at } 25 \mathrm{ng} \text { per tube }(\mathrm{S} / \mathrm{N}=1.5 \text { ) with Jack bean meal urease. }\end{array}$ \\
\hline 100.00 & Copyright 1999 Academic Press. & Copyright 1999 Academic Press. \\
\hline 100.00 & IV. & IV. \\
\hline 100.00 & Copyright 1998 Academic Press. & Copyright 1998 Academic Press. \\
\hline 100.00 & IV. & IV. \\
\hline 100.00 & Biol. & Biol. \\
\hline 100.00 & Copyright 1999 Academic Press. & Copyright 1999 Academic Press. \\
\hline 100.00 & Copyright 1998 Academic Press. & Copyright 1998 Academic Press. \\
\hline 100.00 & Copyright 2000 Academic Press. & Copyright 2000 Academic Press. \\
\hline 100.00 & 1988). & (1988) J. \\
\hline 100.00 & Biol. & Biol. \\
\hline 100.00 & Acad. & Acad. \\
\hline 100.00 & Virol. & Virol. \\
\hline 100.00 & 1995. & (1995) J. \\
\hline 100.00 & Natl. & Natl. $\quad$ \\
\hline 100.00 & Copyright 1999 Academic Press. & Copyright 1999 Academic Press. \\
\hline 100.00 & $\begin{array}{l}\text { The activated glucocorticoid receptor forms a complex with Stat5 and enhances Stat5- } \\
\text { mediated transcriptional induction. }\end{array}$ & $\begin{array}{l}\text { The activated glucocorticoid receptor forms a complex with Stat5 and enhances Stat5- } \\
\text { mediated transcriptional induction. }\end{array}$ \\
\hline 100.00 & Copyright 1999 Academic Press. & Copyright 1999 Academic Press. \\
\hline 100.00 & Chem. & Chem. \\
\hline 100.00 & Appl. & Appl. \\
\hline 100.00 & Copyright 1998 Academic Press. & Copyright 1998 Academic Press. \\
\hline 100.00 & Sci. & Sci. \\
\hline 100.00 & (1992) J. & (1992) J. \\
\hline 100.00 & Acad. & Acad. \\
\hline 100.00 & $\begin{array}{l}\text { Mutational analysis of yeast CEG1 demonstrated that four of the five conserved motifs } \\
\text { are essential for capping enzyme function in vivo. }\end{array}$ & $\begin{array}{l}\text { Mutational analysis of yeast CEG1 demonstrated that four of the five conserved motifs } \\
\text { are essential for capping enzyme function in vivo. }\end{array}$ \\
\hline 100.00 & $\begin{array}{l}\text { We also show that in fusions with the DNA binding domain of GAL4, full activity } \\
\text { requires the entire BHV-alpha TIF, although both amino and carboxyl termini display } \\
\text { some activity on their own. }\end{array}$ & $\begin{array}{l}\text { We also show that in fusions with the DNA binding domain of GAL4, full activity } \\
\text { requires the entire BHV-alpha TIF, although both amino and carboxyl termini display } \\
\text { some activity on their own. }\end{array}$ \\
\hline
\end{tabular}

Table 10: Samples with over $75 \%$ similarity in the BC2GM dataset 


\begin{tabular}{|c|c|c|}
\hline Gene & Position & Input \\
\hline capping enzyme & 88100 & $\begin{array}{l}\text { Mutational analysis of yeast CEG1 demonstrated that four of the five conserved motifs are essential for capping } \\
\text { enzyme function in vivo. }\end{array}$ \\
\hline human IL-4 cytokine gene & 145165 & $\begin{array}{l}\text { Analysis of } 1 \mathrm{Mb} \text { of published sequence from the region of conserved synteny on human chromosome } 5 \mathrm{q} 31-\mathrm{q} 33 \\
\text { identified } 45 \text { gene candidates, including } 35 \text { expressed genes in the human IL- } 4 \text { cytokine gene cluster. }\end{array}$ \\
\hline LH & 1011 & $($ LH P $; 0.05$, LH/FSH P $; 0.01)$. \\
\hline FSH & 1315 & $($ LH P $; 0.05$, LH/FSH P $; 0.01)$ \\
\hline Urease & 05 & $\begin{array}{l}\text { Urease activity, judged as the amount of ammonia production from urea, could be measured at } 25 \mathrm{ng} \text { per tube ( } \mathrm{S} / \mathrm{N} \\
=1.5 \text { ) with Jack bean meal urease. }\end{array}$ \\
\hline cAMP-CRP & 117124 & $\begin{array}{l}\text { The reconstituted RNA polymerases containing the mutant alpha subunits were examined for their response to } \\
\text { transcription activation by cAMP-CRP and the rrnBP1 UP element. }\end{array}$ \\
\hline HIV-2 nef & 5158 & These results demonstrate a specific association of SIV and HIV-2 nef, but not HIV-1 nef, with TCRzeta. \\
\hline HIV-1 nef & 6673 & These results demonstrate a specific association of SIV and HIV-2 nef, but not HIV- 1 nef, with TCRzeta. \\
\hline
\end{tabular}

Table 11: Test samples where the model failed to detect genes, lowering recall, despite the input raw text being an exact match to the training sample 